\title{
Phonon density of states and heat capacity of $\mathrm{La}_{3-x} \mathrm{Te}_{4}$
}

\author{
O. Delaire, ${ }^{1}$ A. F. May, ${ }^{2}$ M. A. McGuire,${ }^{3}$ W. D. Porter, ${ }^{3}$ M. S. Lucas,${ }^{1}$ M. B. Stone,${ }^{1}$ D. L. Abernathy, ${ }^{1}$ V. A. Ravi, ${ }^{4,5}$ \\ S. A. Firdosy, ${ }^{4}$ and G. J. Snyder ${ }^{2}$ \\ ${ }^{1}$ Neutron Scattering Science Division, Oak Ridge National Laboratory, Oak Ridge, Tennessee 37831, USA \\ ${ }^{2}$ California Institute of Technology, Pasadena, California 91125, USA \\ ${ }^{3}$ Materials Science and Technology Division, Oak Ridge National Laboratory, Oak Ridge, Tennessee 37831, USA \\ ${ }^{4}$ Jet Propulsion Laboratory, 4800 Oak Grove Drive, Pasadena, California 91109, USA \\ ${ }^{5}$ Department of Chemical and Materials Engineering, California State Polytechnic University, Pomona, California 91768, USA
}

(Received 25 August 2009; published 19 November 2009)

\begin{abstract}
The phonon density of states (DOS) of $\mathrm{La}_{3-x} \mathrm{Te}_{4}$ compounds $(x=0.0,0.18,0.32)$ was measured at 300, 520, and $780 \mathrm{~K}$, using inelastic neutron scattering. A significant stiffening of the phonon DOS and a large broadening of features were observed upon introduction of vacancies on La sites (increasing $x$ ). Heat-capacity measurements were performed at temperatures $1.85 \leq T \leq 1200 \mathrm{~K}$ and were analyzed to quantify the contributions of phonons and electrons. The Debye temperature and the electronic coefficient of heat capacity determined from these measurements are consistent with the neutron-scattering results, and with previously reported first-principles calculations. Our results indicate that $\mathrm{La}$ vacancies in $\mathrm{La}_{3-x} \mathrm{Te}_{4}$ strongly scatter phonons and this source of scattering appears to be independent of temperature. The stiffening of the phonon DOS induced by the introduction of vacancies is explained in terms of the electronic structure and the change in bonding character. The temperature dependence of the phonon DOS is captured satisfactorily by the quasiharmonic approximation.
\end{abstract}

DOI: $10.1103 /$ PhysRevB.80.184302

PACS number(s): 63.20.-e, 61.05.fg, 65.40.Ba, 72.20.Pa

\section{INTRODUCTION}

The efficiency of thermoelectric materials in converting between thermal and electrical energies is quantified by the dimensionless figure of merit $z T=\alpha^{2} \sigma T / \kappa$, where $\alpha$ is the Seebeck coefficient, $\sigma$ the electrical conductivity, and $\kappa$ the thermal conductivity. ${ }^{1}$ In order to maintain the temperature gradient used to generate power, low thermal conductivity is required, and must be combined with favorable electronic properties (large $\alpha$ and $\sigma$ ) to achieve high conversion efficiency. $\mathrm{La}_{3-x} \mathrm{Te}_{4}$ has the highest known thermoelectric efficiency ( $n$ type) at high temperatures, $z T \simeq 1.2$ at $1300 \mathrm{~K}$. It owes its strong performance to a relatively large Seebeck coefficient and a low-lattice thermal conductivity $\left(\kappa_{l}\right.$ $\leq 1 \mathrm{~W} \mathrm{~m}^{-1} \mathrm{~K}^{-1}$ at $\left.300 \mathrm{~K}\right){ }^{2}$ Lanthanum telluride and other rare-earth chalcogenides with the $\mathrm{Th}_{3} \mathrm{P}_{4}$ structure have been studied over several decades as candidate high-temperature thermoelectrics, ${ }^{3-7}$ as well as for their superconducting properties ${ }^{8-10}$ Early studies of thermoelectric investigations were summarized by Wood. ${ }^{11}$ Measurements of phonons in candidate thermoelectrics are important to gain insight into phonon-scattering mechanisms, and in order to design materials with low $\kappa \cdot{ }^{12-18}$ However, there have been no detailed phonon measurements in $\mathrm{La}_{3-x} \mathrm{Te}_{4}$ to date.

The structure of $\mathrm{La}_{3-x} \mathrm{Te}_{4}$ is that of $\mathrm{Th}_{3} \mathrm{P}_{4}$ (space group $I \overline{4} 3 d)$. In this cubic structure, La cations sit in an irregular coordination polyhedron, with two groups of four Te anions, each forming a tetrahedron around the La. The Te atoms in turn are surrounded by six lanthanums forming a distorted octahedron. ${ }^{19}$ This structure accommodates a large number of vacancies on the cation site, with up to $1 / 9 \mathrm{La}$ atoms vacant, corresponding to $x=1 / 3$. A simple electron count shows that the compound should be metallic for $x=0$ and insulating for $x=1 / 3$, which is indeed observed experimen- tally. The introduction of vacancies therefore offers a way to optimize the electronic properties of the figure of merit. The large number of vacancies that can be controllably achieved in $\mathrm{La}_{3-x} \mathrm{Te}_{4}$ is also thought to reduce the lattice thermal conductivity, $\kappa_{l}$, through scattering of phonons by the point defects. $^{2}$

Here we report neutron-scattering measurements of the phonon density of states (DOS) of $\mathrm{La}_{3-x} \mathrm{Te}_{4}$ for $x$ $=0.0,0.18,0.32$, at $T=300,520$, and $780 \mathrm{~K}$. We also report heat-capacity measurements for the temperature range 1.85 $\leq T \leq 1200 \mathrm{~K}$, and discuss our results in the light of previous transport measurements and electronic-structure calculations.

\section{SYNTHESIS}

Samples were prepared via mechanical alloying as discussed in Ref. 2. This technique produces powder samples from the pure elements near room temperature. The powder samples are then hot pressed (in excess of $1300 \mathrm{~K}$ ) to produce dense pellets, which are more stable in oxidizing conditions and can be characterized for a variety of properties. To produce enough material for the neutron-scattering experiments, several $10 \mathrm{~g}$ batches were produced at each of the desired nominal compositions. Samples of nominal composition $\mathrm{La}_{3} \mathrm{Te}_{4}$ and $\mathrm{La}_{2.82} \mathrm{Te}_{4}$ were the result of four $10 \mathrm{~g}$ loads, which were hot pressed in $20 \mathrm{~g}$ batches to yield two separate cylindrical pucks (for each nominal composition). For the $\mathrm{La}_{2.68} \mathrm{Te}_{4}$ sample, only one $20 \mathrm{~g}$ puck was obtained due to processing difficulty. The phase purity of all samples was examined with x-ray diffraction. The $\mathrm{La}_{3} \mathrm{Te}_{4}$ and $\mathrm{La}_{2.68} \mathrm{Te}_{4}$ samples contained a single phase but the diffraction patterns for $\mathrm{La}_{2.82} \mathrm{Te}_{4}$ indicated the presence of a small 
TABLE I. Room-temperature transport properties of $\mathrm{La}_{3-x} \mathrm{Te}_{4}$ samples (values in parentheses are standard deviations).

\begin{tabular}{lccccc}
\hline \hline Sample & $\begin{array}{c}\alpha \\
\left.(\mu \mathrm{V} \mathrm{K})^{-1}\right)\end{array}$ & $\begin{array}{c}\rho \\
(\mathrm{m} \mathrm{cm})\end{array}$ & $\begin{array}{c}D_{T} \\
\left(\mathrm{~mm}^{2} \mathrm{~s}^{-1}\right)\end{array}$ & $\begin{array}{c}\kappa \\
\left(\mathrm{W} \mathrm{m}^{-1} \mathrm{~K}^{-1}\right)\end{array}$ & $\begin{array}{c}\kappa_{l} \\
\left(\mathrm{~W} \mathrm{~m}^{-1} \mathrm{~K}^{-1}\right)\end{array}$ \\
\hline $\mathrm{La}_{3} \mathrm{Te}_{4}$ & $25(1)$ & $0.34(0.03)$ & 2.45 & 3.2 & 1.0 \\
$\mathrm{La}_{2.82} \mathrm{Te}_{4}$ & $51(4)$ & $4.5(1.0)$ & 0.91 & 1.2 & 1.0 \\
$\mathrm{La}_{2.68} \mathrm{Te}_{4}$ & $307(2)$ & $6.5 \times 10^{6}$ & 0.60 & 0.65 & 0.65 \\
\hline \hline
\end{tabular}

amount of $\mathrm{La}_{2} \mathrm{O}_{2} \mathrm{Te}$ impurity (the impurity phase fraction was estimated to be about $2.5 \%$ ).

Room-temperature transport properties were characterized and the results are given in Table I. The standard deviations provided are representative of the degree to which the samples utilized for neutron scattering are homogeneous. Therefore, the transport data in Table I imply that the sample of nominal composition $\mathrm{La}_{2.82} \mathrm{Te}_{4}$ contains the largest amount of inhomogeneity, likely related to the oxidation, while the other two samples contain little inhomogeneity.

From the transport properties listed in Table I, and the data in Ref. 2, it is inferred that the nominal compositions are accurate descriptions of the true compositions. For instance, the large magnitude of the Seebeck coefficient $(\alpha)$ and electrical resistivity $(\rho)$ for $\mathrm{La}_{2.68} \mathrm{Te}_{4}$ strongly suggest that the composition is near the insulating limit of $\mathrm{La}_{2.667} \mathrm{Te}_{4}$. Some discrepancy is observed in the $\mathrm{La}_{2.82} \mathrm{Te}_{4}$ data: the $\alpha$ data implies the nominal composition is roughly correct but the $\rho$ and thermal diffusivity $\left(D_{T}\right)$ data suggest the composition is closer to $\mathrm{La}_{2.70} \mathrm{Te}_{4}$. We are inclined to believe the Seebeck coefficient data for this particular discussion because $\alpha$ is less influenced by grain-boundary resistances than are $\rho$ and $D_{T}$, which can be significantly altered by small amounts of oxide at the grain boundaries. Therefore, we conclude the nominal compositions are an adequate description of the actual composition while noting that sample $\mathrm{La}_{2.82} \mathrm{Te}_{4}$ is less homogeneous than the others and may exhibit additional grain-boundary resistance.

Also shown in Table $I$ is the thermal conductivity $(\kappa$ $\left.=C_{P} D_{T} d\right)$, which was obtained from the geometric density $(d)$, the measured heat capacity $\left(C_{P}\right)$ (shown in Table II), and the measured thermal diffusivity $\left(D_{T}\right)$. The values of $D_{T}$

TABLE II. Summary of low- $T$ heat-capacity measurements.

\begin{tabular}{lccc}
\hline \hline Sample & $\begin{array}{c}\gamma_{\mathrm{el}} \\
( \pm 5 \%) \\
(\mathrm{mJ} / \mathrm{mol} \text { at. K})\end{array}$ & $\begin{array}{c}\theta_{\mathrm{D}} \\
( \pm 5 \%) \\
(\mathrm{K})\end{array}$ & $\begin{array}{c}C_{P}(300 \mathrm{~K}) \\
( \pm 1 \%) \\
\left(k_{\mathrm{B}} / \mathrm{at} .\right)\end{array}$ \\
\hline $\mathrm{La}_{3} \mathrm{Te}_{4}(H=0 \mathrm{~T}){ }^{\mathrm{a}}$ & 2.65 & 195 & 3.13 \\
$\mathrm{La}_{3} \mathrm{Te}_{4}(H=12 \mathrm{~T})$ & 2.91 & 184 & \\
$\mathrm{La}_{2.82} \mathrm{Te}_{4}(\mathrm{~s} 1)$ & 0.43 & 210 & 3.11 \\
$\mathrm{La}_{2.82} \mathrm{Te}_{4}(\mathrm{~s} 2)$ & 0.30 & 210 & 3.10 \\
$\mathrm{La}_{2.68} \mathrm{Te}_{4}(\mathrm{~s} 1)$ & $<1 \mathrm{~b}$ & 235 & 3.04 \\
$\mathrm{La}_{2.68} \mathrm{Te}_{4}(\mathrm{~s} 2)$ & $<1 \mathrm{~b}$ & 238 & 3.04 \\
\hline
\end{tabular}

${ }^{a}$ Fit performed above $4 \mathrm{~K}$. Other fits were to data below $2.5 \mathrm{~K}$.

${ }^{b}$ Expected to be an upper bound due to contributions from magnetic impurities. were obtained using a NETZSCH LFA 457 flash diffusivity apparatus operated with samples under vacuum. The dependence of $\kappa$ on composition is primarily the result of a change in the electronic contribution $\left(\kappa_{e}\right)$, though changes in the lattice contribution $\left(\kappa_{l}\right)$ also occur. As in Ref. 2, the values of $\kappa_{l}$ were obtained by assuming acoustic-phonon-scattering limits the carrier mobility. These trends are expected, as a large $\kappa_{e}$ exists for the low- $\rho$ samples, and a decrease in $\kappa_{l}$ is expected with increasing vacancy concentration (point-defect scattering of phonons). In a prior publication, ${ }^{2}$ a smaller value of $C_{P}$ was utilized, which resulted in a low $\kappa_{l}$ $=0.4 \mathrm{~W} \mathrm{~m}^{-1} \mathrm{~K}^{-1}$ for samples near the $\mathrm{La}_{3} \mathrm{Te}_{4}$ composition (at moderate $T$ ). This was believed to be due to strong electron-phonon coupling, which is responsible for the superconductivity at low $T$. However, the current correction to $C_{P}$ suggests that electron-phonon interactions do not yield such significant reductions of $\kappa_{l}$ in $\mathrm{La}_{3-x} \mathrm{Te}_{4}$.

\section{INELASTIC NEUTRON SCATTERING}

\section{A. Experimental method}

Inelastic neutron-scattering (INS) spectra were measured using the ARCS time-of-flight chopper spectrometer at the Spallation Neutron Source, at Oak Ridge National Laboratory. The samples were encased in a thin-walled cylindrical Al cell, $12 \mathrm{~mm}$ in diameter. For measurements at low temperatures, the sample holder was cooled by a closed-cycle $\mathrm{He}$ refrigerator, while the high-temperature measurements were performed with the sample assembly in a low-background furnace, which was kept under high vacuum during all measurements. No oxidation could be seen on the samples after the measurements. We used an incident neutron energy $E_{i}$ $=40 \mathrm{meV}$. The energy resolution (full width at half maximum) was about $1.1 \mathrm{meV}$ at $15 \mathrm{meV}$ neutron energy loss, increasing to about $1.9 \mathrm{meV}$ at the elastic line. The empty $\mathrm{Al}$ sample container was measured in identical conditions at all temperatures.

All spectra were normalized by the total incident flux and corrected for detector efficiency using a vanadium standard. In all cases, the spectrum from the empty sample container was subtracted. The powder data were analyzed in the incoherent-scattering approximation, which works well when the data are averaged over a large range of momentum transfers, $Q$. In this study, the momentum transfer was integrated over the range $1.5 \leq Q \leq 7.0 \AA^{-1}$, corresponding to an averaging over phonon dispersions in many Brillouin zones. The incoherent-inelastic neutron scattering cross section is proportional to the incoherent-scattering function, which for 
single-phonon creation processes (corresponding to neutron energy loss) in a monatomic cubic material is given by

$$
S(Q, E)=\frac{\hbar^{2} Q^{2}}{4 M} \exp (-2 W) \frac{g(E)}{E}[n(E)+1],
$$

where $Q$ and $E$ are the momentum and energy transfer to the sample, $M$ and $\exp (-2 W)$ are the mass and Debye-Waller factor, respectively, and $n(E)$ is the Bose-Einstein occupation factor. ${ }^{20,21}$ The elastic peak was removed, and the data below $3 \mathrm{meV}$ were extrapolated using a Debye model. An iterative procedure was used to correct for the effect of multiphonon scattering. ${ }^{22}$

In a polyatomic material, different elements have different factors $\sigma / M$, the modes corresponding to the elements of higher scattering efficiency are overemphasized compared to those of elements with lesser neutron-scattering efficiency, resulting in a neutron-weighted phonon DOS, $g_{\mathrm{NW}}(E)$,

$$
g_{\mathrm{NW}}(E)=\sum_{i} c_{i}\left(\sigma_{i} / M_{i}\right) g_{i}(E) \exp \left(-2 W_{i}\right)
$$

where $i$ runs over the different elements, $c_{i}$ is the atomic concentration, $g_{i}$ the partial phonon DOS of species $i$, $\exp ($ $\left.-2 W_{i}\right)$ is the Debye-Waller factor, and $\sigma_{i}$ and $M_{i}$ are the neutron cross section and atomic mass. Because we average over the coherent as well as incoherent phonon-scattering intensity, the total neutron cross sections are used in Eq. (2). In the case where the partial phonon DOS curves for the different species are similar, there is little overall effect from neutron weighting on the shape of the phonon DOS. The values of $\sigma / M$ for $\mathrm{La}$ and $\mathrm{Te}$ are $6.95 \times 10^{-2}$ and 3.39 $\times 10^{-2} \mathrm{~b} / \mathrm{amu}$, respectively. Thus, the modes involving large motions of $\mathrm{La}$ atoms are overemphasized in the measured phonon DOS. However, the mass ratio is close to unity, $M_{\mathrm{La}} / M_{\mathrm{Te}}=1.09$, so the partial phonon DOS of La and Te should be similar (and so should the Debye-Waller factors), limiting greatly the effect of neutron weighting on the measured phonon DOS. In the following, the measured $g(E)$ results refer to the neutron-weighted phonon DOS.

\section{B. Composition dependence of the phonon DOS}

The phonon DOS of the three compounds at $300 \mathrm{~K}$ are shown in Fig. 1. We observe well-defined phonon peaks, with a low-energy peak at $\sim 8 \mathrm{meV}$, and two optical peaks at 12 and $16 \mathrm{meV}$ in $\mathrm{La}_{3} \mathrm{Te}_{4}$, which coalesce into a broader peak at $\sim 15 \mathrm{meV}$ in the samples with vacancies. Considering that there are 28 atoms in the unit cell, each of these peaks accounts for a large number of phonon branches. The phonon cutoff for $\mathrm{La}_{3} \mathrm{Te}_{4}$ is sharp with an energy $E_{\text {cutoff }}$ $=18.5 \mathrm{meV}$. The compounds with La vacancies have stiffer (higher-energy) phonons. The phonon cutoff energy is about $1.5 \mathrm{meV}$ higher in $\mathrm{La}_{2.68} \mathrm{Te}_{4}$ than in $\mathrm{La}_{3} \mathrm{Te}_{4}$. The stiffening affects the full phonon spectrum, however the relative amount of stiffening $\Delta E / E$ is larger at the low-energy acoustic peak (about $12 \%$ at $8 \mathrm{meV}$ ) than at the optical peaks (about $5 \%$ at $15 \mathrm{meV}$ ). From the phonon DOS, we calculate the Debye temperature using the relation $\theta_{\mathrm{D}}=4 / 3\langle E\rangle / k_{\mathrm{B}}$, where $\langle E\rangle=\int E g(E) d E .^{23} \mathrm{We}$ find: $\theta_{\mathrm{D}}=173,182$, and $182 \mathrm{~K}$ for $\mathrm{La}_{3} \mathrm{Te}_{4}, \mathrm{La}_{2.82} \mathrm{Te}_{4}$, and $\mathrm{La}_{2.68} \mathrm{Te}_{4}$, respectively.

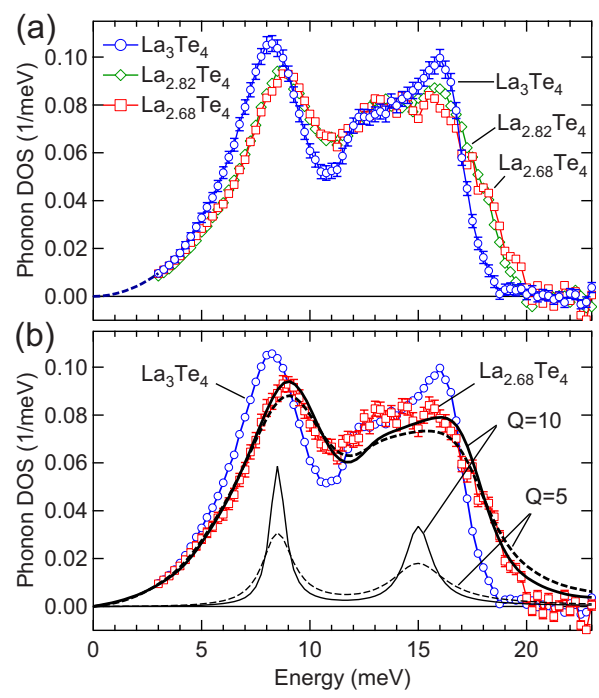

FIG. 1. (Color online) (a) Neutron-weighted phonon DOS of $\mathrm{La}_{3-x} \mathrm{Te}_{4}$ at $300 \mathrm{~K}$, measured by inelastic neutron scattering $\left(E_{i}\right.$ $=40 \mathrm{meV}$ ). Error bars represent uncertainties from counting statistics of the measurement. (b) The thick curves labeled $\mathcal{Q}=5$ and $\mathcal{Q}=10$ were obtained by convolving the DOS for $\mathrm{La}_{3} \mathrm{Te}_{4}$ with a damped oscillator linewidth of corresponding quality factor $\mathcal{Q}$ and applying a shift of the energy axis $E^{\prime}=(1.15+(1.05-1.15)$ $\times E / 16.0) \times E$. The thin curves at the bottom illustrate the phonon line shapes $\mathcal{L}_{\mathcal{Q}, E_{0}}(E)$ at $E_{0}=8.5$ and $15 \mathrm{meV}$, for $\mathcal{Q}=5$ and $10 \mathrm{meV}$.

We also observe a considerable broadening of the phonon DOS upon introduction of vacancies, especially for the optical-phonon peaks, between 11 and $17 \mathrm{meV}$. We expect this broadening to be due to scattering of phonons by the vacancies in the lattice, and to correlate to the reduced thermal conductivity of the system with increasing vacancies. The greater broadening of the high-energy modes makes physical sense, as point defects scatter short wavelength, high-energy phonons more effectively than long-wavelength phonons.

The broadening of the phonon DOS is well reproduced using a damped harmonic-oscillator line shape ${ }^{20}$

$$
\mathcal{L}_{\mathcal{Q}, E_{0}}(E)=\frac{1}{\pi \mathcal{Q} E_{0}} \frac{1}{\left(\frac{E_{0}}{E}-\frac{E}{E_{0}}\right)^{2}+\frac{1}{\mathcal{Q}^{2}}},
$$

where $\mathcal{Q}$ is the quality factor of the damped oscillator. Physically, the quality factor $\mathcal{Q}$ quantifies the number of oscillations the phonon of energy $E_{0}$ completes before decaying through interactions with defects, other phonons, or electrons. Using the phonon DOS of the material free of vacancies $\left(\mathrm{La}_{3} \mathrm{Te}_{4}\right)$ as reference, we can convolute the DOS with the broadening function and estimate the quality factor $\mathcal{Q}$ representative of phonon lifetimes in the material with the most vacancies, $\mathrm{La}_{2.68} \mathrm{Te}_{4}$. We estimated values of $\mathcal{Q}$ of about 10. The result of convoluting the phonon DOS of $\mathrm{La}_{3} \mathrm{Te}_{4}$ at $300 \mathrm{~K}$ with this damped oscillator linewidth, and rescaling the energy axis, is shown in Fig. 1(b). As can be seen, the broadening and stiffening of the phonon energies observed in the compound with most vacancies $(x=0.32)$ is 

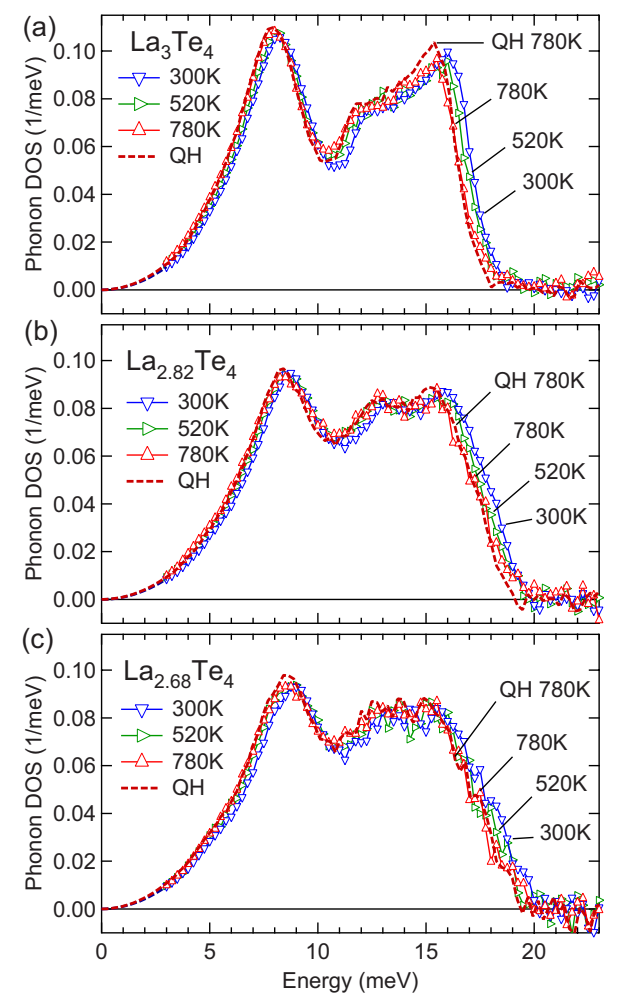

FIG. 2. (Color online) Neutron-weighted phonon DOS curves measured at 300, 520, and $780 \mathrm{~K}$ for (a) $\mathrm{La}_{3} \mathrm{Te}_{4}$, (b) $\mathrm{La}_{2.82} \mathrm{Te}_{4}$, and (c) $\mathrm{La}_{2.68} \mathrm{Te}_{4}$. Dashed red lines in all panels $(\mathrm{QH} 780 \mathrm{~K})$ correspond to the quasiharmonic prediction for the phonon DOS at $780 \mathrm{~K}$, given the phonon DOS at $300 \mathrm{~K}$, and a softening of phonon energies $E_{780}=0.96 \times E_{300}$.

well reproduced for $\mathcal{Q}=10$. A better fit might be obtained by using a larger broadening (lower $\mathcal{Q}$ ) for optical modes, and a narrower value for acoustic modes, but it is difficult to justify where the separation should be done, thus we preferred to use a single $\mathcal{Q}$ parameter.

\section{Temperature dependence of the phonon DOS}

The temperature dependence of the phonon DOS for all three samples is shown in Fig. 2. Increasing temperature from 300 to $750 \mathrm{~K}$ leads to a gradual softening of the DOS for all three compositions. The amount of softening is comparable in all three cases. This softening of phonons with increasing temperature is the behavior typically observed in most materials and is often rationalized within the quasiharmonic $(\mathrm{QH})$ model. In this model, phonon frequencies depend on temperature solely through the change in volume of the system. The relative change in phonon energy is related to the relative change in volume through the Grüneisen parameter, $\gamma_{\mathrm{G}}=-d \ln E / d \ln V$. A common simplification is to consider the average Grüneisen parameter for all phonon modes, $\bar{\gamma}_{\mathrm{G}}=-d \ln \langle E\rangle / d \ln V$. This average, or thermodynamic, Grüneisen parameter can be expressed in terms of standard thermodynamic quantities, ${ }^{23}$

$$
\bar{\gamma}_{\mathrm{G}}=\frac{3 \alpha V B_{T}}{C_{V}}=\frac{3 \alpha V B_{S}}{C_{P}},
$$

where $\alpha$ is the linear coefficient of thermal expansion, $B_{T}$ (respectively, $B_{S}$ ) is the isothermal (respectively, isentropic)

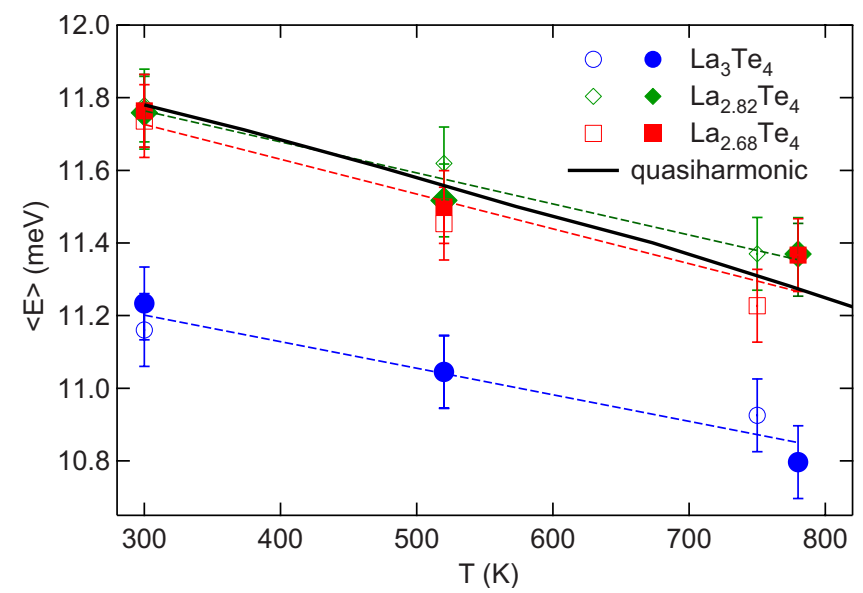

FIG. 3. (Color online) Temperature dependence of the average phonon energy, $\langle E\rangle$, of $\mathrm{La}_{3-x} \mathrm{Te}_{4}$, determined from the phonon DOS. Closed and open markers are for measurements performed with incident neutron energies $E_{i}=40$ and $80 \mathrm{meV}$, respectively. The thick dark line is the expected quasiharmonic dependence of $\langle E\rangle$ for $\mathrm{La}_{2.82} \mathrm{Te}_{4}$ based on our dilatometry measurement and a Grüneisen parameter $\bar{\gamma}_{\mathrm{G}}=1.76$.

bulk modulus, and $C_{V}$ (respectively, $C_{P}$ ) is the heat capacity at constant volume (respectively, constant pressure). As $\bar{\gamma}_{\mathrm{G}}$ is typically positive, one expects a decrease in phonon frequencies with increasing temperature, following thermal expansion. The phonon DOS of all three compositions appear in good agreement with this description. In particular, the shift in energy of the modes observed at any given temperature is proportional to the energy of each mode, $\Delta E \propto E$, as in the $\mathrm{QH}$ model. This can also be tested by performing a scaling of the energy axis. Scaling the energy so that $E_{780}=0.96$ $\times E_{300}$ brings the phonon DOS measured at $300 \mathrm{~K}$ in excellent agreement with the DOS measured at $780 \mathrm{~K}$ for all three compositions, as can be seen in Fig. 2 (dashed lines).

The temperature dependence of the average phonon energy, $\langle E\rangle$, is shown in Fig. 3. In the three samples, $\langle E\rangle$ decreases with $T$, and the dependence appears linear within our experimental error bars. The dependence of $\langle E\rangle$ on $T$ according to the $\mathrm{QH}$ model was calculated using our results for the thermal expansion of two slices of nominal compositions $\mathrm{LaTe}_{1.40}$ and $\mathrm{LaTe}_{1.425}$, measured with dilatometry (both compositions gave the same results). A Grünesein parameter $\bar{\gamma}_{\mathrm{G}}=1.76$ was estimated. ${ }^{2}$ The resulting curve (thick dark line in Fig. 3) is in very good agreement with the measured average phonon energy, indicating that the material with vacancies follows the quasiharmonic behavior. This conclusion can be surprising, considering that introducing vacancies results in a considerable reduction in lattice thermal conductivity and a broadening of the phonon DOS. More will be said about this in the discussion.

\section{CALORIMETRY}

\section{A. Low-temperature heat capacity}

Constant-pressure heat-capacity measurements were performed on two small (10-20 mg) pieces of each sample, 

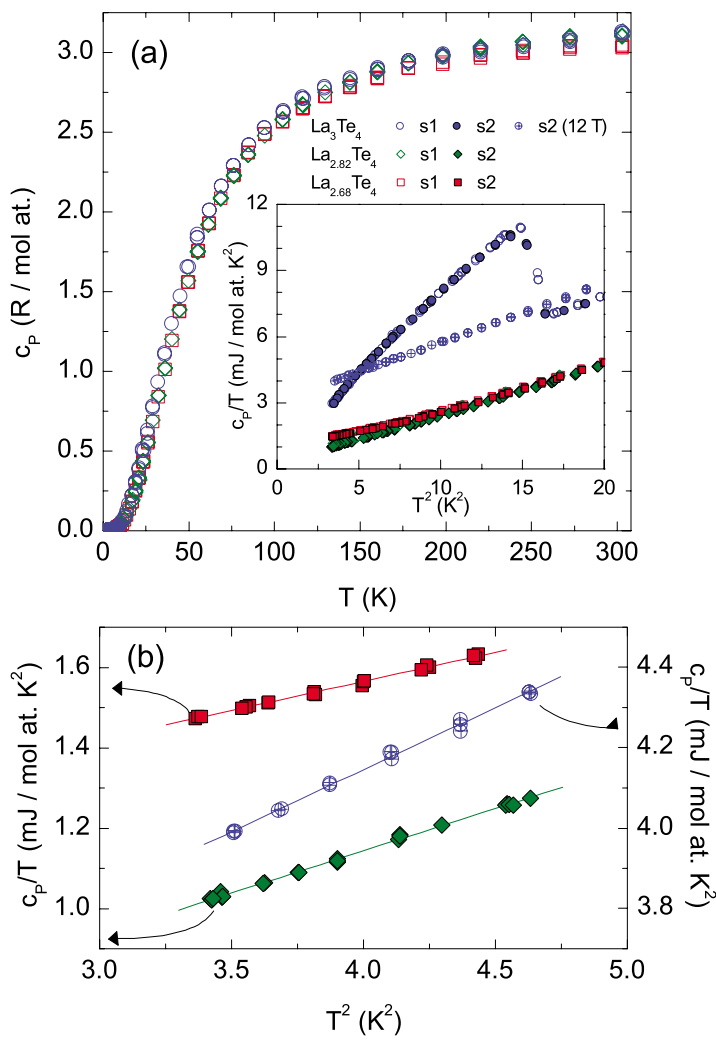

FIG. 4. (Color online) (a) Heat-capacity data for $\mathrm{La}_{3} \mathrm{Te}_{4}$, $\mathrm{La}_{2.82} \mathrm{Te}_{4}$, and $\mathrm{La}_{2.68} \mathrm{Te}_{4}$, measured with Quantum Design PPMS apparatus. The inset shows $C_{P} / T$ vs $T^{2}$ for $T<4.5 \mathrm{~K}$. (b) Fits of $C_{P} / T$ vs $T^{2}$ to obtain $\gamma_{\mathrm{el}}$ and $\theta_{\mathrm{D}}$ in Table II (see text).

denoted here as s1 and s2, using a Quantum Design physical property measurement system (PPMS). Data were collected at $T \sim 1.85-300 \mathrm{~K}$ in zero applied magnetic field for all samples with the exception of $\mathrm{La}_{3} \mathrm{Te}_{4}$ sample 2 (s2), for which data were collected over the temperature range of $1.86-10 \mathrm{~K}$ in zero applied field and at $12 \mathrm{~T}$. Results are shown in Fig. 4. The temperature data were fit to extract the coefficient of electronic specific heat, $\gamma_{\mathrm{el}}$, and the Debye temperature, $\theta_{\mathrm{D}}$, using the relationship: $C_{P} / T=\gamma_{\mathrm{el}}+\beta T^{2}$, where $\beta=12 \pi^{4} /\left(5 \theta_{\mathrm{D}}^{3}\right) .{ }^{23}$ Results are given in Table II. We note that the value of $210 \mathrm{~K}$ for $\theta_{\mathrm{D}}$ of $\mathrm{La}_{2.82} \mathrm{Te}_{4}$ determined from calorimetry is in excellent agreement with the value of $208 \mathrm{~K}$ determined from sound velocity measurements in $\mathrm{La}_{2.81} \mathrm{Te}_{4} \cdot{ }^{2}$ Our heat-capacity data for $\mathrm{La}_{3} \mathrm{Te}_{4}$ are in good agreement with results reported by Mitarov et al. ${ }^{24}$ who found that $C_{P}$ crosses the Dulong-Petit limit of $3.0 k_{\mathrm{B}} /$ atom at $250 \mathrm{~K}$ (we find $C_{P}=3.04 k_{\mathrm{B}}$ /atom at this temperature).

The stoichiometric compound $\mathrm{La}_{3} \mathrm{Te}_{4}$ is superconducting below $4 \mathrm{~K}$, and the superconducting transition is clearly observed in our $C_{P}$ data, confirming the quality of the sample. For the data obtained at $H=0 \mathrm{~T}$, only the part of the $C_{P}$ curve above $T_{c}$ was used to determine $\gamma_{\mathrm{el}}$ and $\theta_{\mathrm{D}}$. In an applied magnetic field of $H=12 \mathrm{~T}$, the superconducting transition was suppressed to $T_{c}<1.8 \mathrm{~K}$, and the heat-capacity data were analyzed in the same temperature range as the nonsuperconducting samples, $1.8<T<2.5 \mathrm{~K}$. Data are shown in Fig. 4(b).

As summarized in Table II, the samples with higher vacancy concentrations have larger values of $\theta_{\mathrm{D}}$. This is in agreement with the phonon stiffening seen in our neutronscattering measurements. However, the change in $\theta_{\mathrm{D}} \mathrm{ob}-$ tained from fits of $C_{P}$ is larger than observed in the measured phonon DOS. We believe that this discrepancy arises from $C_{P}$ fits being performed with very low-temperature data $(T$ $<5 \mathrm{~K})$. This is, however, required for $C_{P} / T$ to depend linearly on $T^{2}$. At such low temperatures, only the lowestenergy phonons are thermally populated, so these estimates of $\theta_{\mathrm{D}}$ are mostly sensitive to the acoustic modes (hence the good agreement with the value derived from sound velocities). On the other hand, the values of $\theta_{\mathrm{D}}$ estimated from the phonon DOS used the entire spectra. As was pointed out above, the acoustic modes stiffen more than the rest of the phonon DOS upon introduction of La vacancies. Thus, it is expected that estimates of $\theta_{\mathrm{D}}$ from PPMS data should show a larger stiffening than those from $\langle E\rangle$ measured with INS.

Also, it is possible that the temperature dependence of phonons in $\mathrm{La}_{3} \mathrm{Te}_{4}$ below $300 \mathrm{~K}$ differs from that of the materials with vacancies since the electron-phonon coupling must be strong in the stoichiometric material. In this regard, it is worth mentioning that an anomalous temperature dependence of the $C_{11}-C_{12}$ elastic constant between 20 and $200 \mathrm{~K}$ was reported in Ref. 25. Tikhonov et al. reported Debye temperatures at $77 \mathrm{~K}$ of $183 \pm 2 \mathrm{~K}$ for $\mathrm{La}_{3} \mathrm{Te}_{4}$ and $192 \pm 2 \mathrm{~K}$ for $\mathrm{La}_{2} \mathrm{Te}_{3}$, from an analysis of heat-capacity measurements. $^{26}$ Their results are in excellent agreement with our result for $\mathrm{La}_{3} \mathrm{Te}_{4}$ at very low $T$ but somewhat smaller than our result in the case of $\mathrm{La}_{2} \mathrm{Te}_{3}$. The value of $\theta_{\mathrm{D}}$ $(205 \mathrm{~K})$ reported by Westerholt et al. for $\mathrm{La}_{3} \mathrm{Te}_{4}$ is somewhat larger than our result and that of Tikhonov et al. ${ }^{10}$

We also find that $\gamma_{\mathrm{el}}$ is significantly suppressed by the introduction of vacancies, corresponding to a large reduction in the electronic density of states at the Fermi level, $N\left(E_{\mathrm{F}}\right)$, since $\gamma_{\mathrm{el}} \propto N\left(E_{\mathrm{F}}\right)$. In the case of $\mathrm{La}_{2.82} \mathrm{Te}_{4}$, we find a reduction in $\gamma_{\mathrm{el}}$ by a factor of $\sim 8$ compared to $\mathrm{La}_{3} \mathrm{Te}_{4}$. In the case of $\mathrm{La}_{2.68} \mathrm{Te}_{4}$, the heat capacity presents a slight upturn at the lowest temperatures [see inset in Fig. 4(a)], which is possibly due to a small amount of magnetic impurities. Magnetization measurements at $5 \mathrm{~K}$ (not shown) reveal a small ferromagnetic component with a saturation moment per atom of about $0.006 \mu_{\mathrm{B}}$ and $0.001 \mu_{\mathrm{B}}$ for $\mathrm{La}_{2.68} \mathrm{Te}_{4}$ and $\mathrm{La}_{2.82} \mathrm{Te}_{4}$, respectively. Likely sources of magnetism in these samples are contamination of the La starting material with Ce or other magnetic rare earths, and Fe incorporation from the stainless steel ball milling containers. For comparison, these saturation moments are equivalent to about 0.3 and 0.05 at. $\% \mathrm{Fe}$ for $\mathrm{La}_{2.68} \mathrm{Te}_{4}$ and $\mathrm{La}_{2.82} \mathrm{Te}_{4}$, respectively. This observations may explain why the value of gamma derived for $\mathrm{La}_{2.82} \mathrm{Te}_{4}$ is larger than that for $\mathrm{La}_{2.68} \mathrm{Te}_{4}$.

The significantly smaller values of gamma observed in the La-deficient materials relative to $\mathrm{La}_{3} \mathrm{Te}_{4}$ are compatible with first-principles calculations of the electronic density of states $N(E)$ and rigid-band calculations of the composition dependence of the Fermi level, $E_{\mathrm{F}}$ (see below). ${ }^{27}$

\section{B. High-temperature heat capacity and thermal expansion}

Heat-capacity measurements were conducted on $\mathrm{La}_{3} \mathrm{Te}_{4}$ for $400 \leq T \leq 1200 \mathrm{~K}$, using a Netzsch 404c differential 


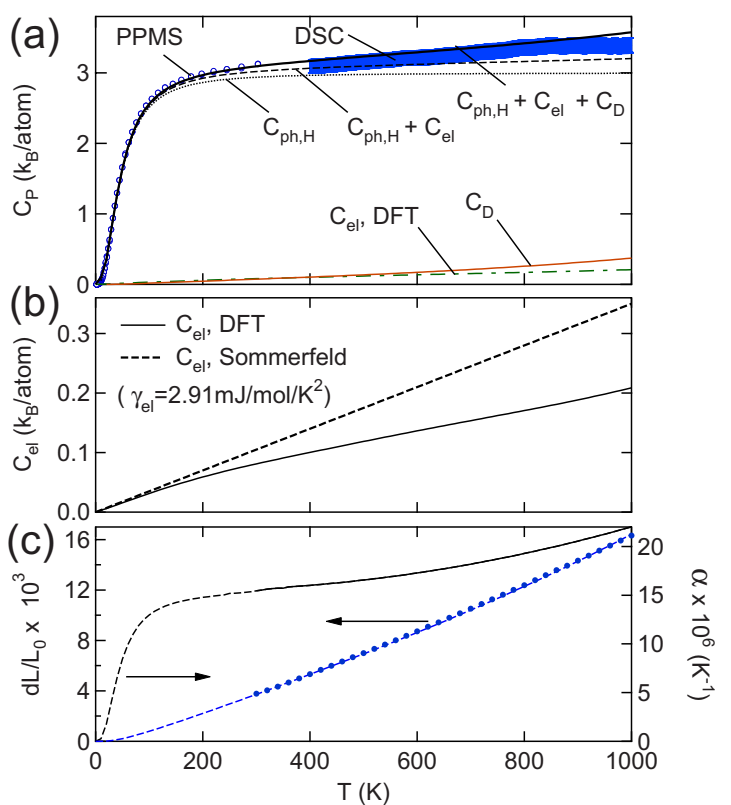

FIG. 5. (Color online) (a) $\mathrm{La}_{3} \mathrm{Te}_{4} C_{P}$ curves, measured at low (PPMS) and high temperatures (DSC), and calculated $C_{P}$ components from harmonic phonons $\left(C_{\mathrm{ph}, \mathrm{H}}\right)$, electrons $\left(C_{\mathrm{el}}\right)$, and dilation of the lattice $\left(C_{\mathrm{D}}\right)$ (see text). (b) Detail of electronic heat capacity calculated from density-functional theory results reported in Ref. 27, and Sommerfeld approximation $C_{\mathrm{el}}=\gamma_{\mathrm{el}} T$, with experimental $\gamma_{\mathrm{el}}$ from PPMS measurements. (c) Measured thermal expansion $d L / L_{0}$ of $\mathrm{La}_{2.86} \mathrm{Te}_{4}$ (markers), extrapolated at low temperatures using measured heat capacity (see text), and linear thermal-expansion coefficient, $\alpha$.

scanning calorimeter (DSC). The measurements were performed with the samples inside Pt crucibles with alumina liners under an ultrapure Ar purge gas cycled through a $\mathrm{Ti}$ gettering furnace. The scans were performed after a careful evacuation of the sample chamber. The temperature scan rate was $20 \mathrm{~K} / \mathrm{min}$. Results are shown in Fig. 5(a).

Thermal expansion was measured between 300 and 1000 $\mathrm{K}$ on a sample of $\mathrm{La}_{2.86} \mathrm{Te}_{4}$ (nominal composition), using a Netzsch DIL 402c dilatometer. Measurements were performed with a heating rate of $2 \mathrm{~K} / \mathrm{min}$, under an $\mathrm{Ar}-7 \% \mathrm{H}_{2}$ atmosphere, flowing at $50 \mathrm{ml} / \mathrm{min}$. A sapphire standard was used for calibration. The linear thermal-expansion coefficient, $\alpha=1 / L_{0} d L / d T$, was obtained from the dilatometry data in the range $300 \leq T \leq 1000 \mathrm{~K}$, and was extrapolated at lower temperatures, using the approximate proportionality of thermal expansion and heat capacity (Grüneisen's rule). ${ }^{23}$ The results are shown in Fig. 5(c). Additional measurements on a sample of $\mathrm{La}_{2.81} \mathrm{Te}_{4}$ gave nearly identical results (within $2 \%)$.

The individual contributions of phonons, electrons, and of the effect of thermal expansion, to the heat capacity of $\mathrm{La}_{3} \mathrm{Te}_{4}$ were computed as follows. The phonon entropy $S_{\mathrm{ph}}$ is obtained from the phonon DOS $g(E)$, as ${ }^{28}$

$$
S_{\mathrm{ph}}=3 k_{\mathrm{B}} \int d E g(E)[(n+1) \ln (n+1)-n \ln n],
$$

where $n=\left\{\exp \left[E /\left(k_{\mathrm{B}} T\right)\right]-1\right\}^{-1}$. The contribution of harmonic phonons, $C_{\mathrm{ph}, \mathrm{H}}$, can be obtained from Eq. (5), as
$C_{\mathrm{ph}, \mathrm{H}}=T d S_{\mathrm{ph}} / d T$. It was derived from the phonon DOS measured at $300 \mathrm{~K}$ with neutron scattering. The contribution of phonons in the harmonic regime does not depend on volume, so the constant-pressure and constant-volume results are identical: $\left.C_{\mathrm{ph}, \mathrm{H}}\right|_{P}=\left.C_{\mathrm{ph}, \mathrm{H}}\right|_{V}$.

The electron entropy at finite temperature is associated with the availability of unoccupied states above the Fermi level and is given by $^{28}$

$$
S_{\mathrm{el}}=-k_{\mathrm{B}} \int d E N(E)[(1-f) \ln (1-f)+f \ln f],
$$

where $f$ is the Fermi-distribution function and $N(E)$ is the electron density of states. ${ }^{28}$ The heat-capacity contribution from electrons at constant volume, $C_{\mathrm{el}}=T d S_{\mathrm{el}} / d T$, was computed from the electronic DOS obtained from first-principles calculations by May et $a .^{27}$

The effect of thermal expansion on the phonon and the electron heat capacities can be derived from the thermal expansion of the system. The effect of lattice dilation on the total heat capacity, $C_{\mathrm{D}}$, can be obtained from

$$
C_{\mathrm{D}}(T)=C_{\mathrm{el}, \mathrm{D}}+C_{\mathrm{ph}, \mathrm{D}}=9 B v \alpha^{2} T,
$$

where $B$ is the isothermal bulk modulus, $\alpha$ is the linear coefficient of thermal expansion, and $v$ is the specific volume (all dependent on temperature). ${ }^{28}$ The effect of dilation is mostly phononic in origin, as $C_{\mathrm{ph}}$ is much larger than $C_{\mathrm{el}}$. The phonon heat capacity can be separated into a harmonic component, $C_{\mathrm{ph}, \mathrm{H}}$, a component representing the effect of dilation, $C_{\mathrm{ph}, \mathrm{D}}$, and a purely anharmonic component associated with the temperature dependence of the vibrations at a constant volume, $C_{\mathrm{ph}, \mathrm{A}}$,

$$
C_{\mathrm{ph}}=C_{\mathrm{ph}, \mathrm{H}}+C_{\mathrm{ph}, \mathrm{D}}+C_{\mathrm{ph}, \mathrm{A}} .
$$

The sum of the harmonic and dilation components is usually called the quasiharmonic term,

$$
C_{\mathrm{ph}, \mathrm{QH}}=C_{\mathrm{ph}, \mathrm{H}}+C_{\mathrm{ph}, \mathrm{D}},
$$

and with this notation

$$
C_{\mathrm{ph}}=C_{\mathrm{ph}, \mathrm{QH}}+C_{\mathrm{ph}, \mathrm{A}} .
$$

Having computed $C_{\mathrm{ph}, \mathrm{H}}, C_{\mathrm{el}}$, and $C_{\mathrm{D}}$, we can compare their sum to the total heat capacity that we obtained from PPMS and DSC measurements. The only components that are not explicitly included in the sum are the anharmonic phonon term, $C_{\mathrm{ph}, \mathrm{A}}$, and a possible term for the electron-phonon coupling effect, $C_{\mathrm{el}-\mathrm{ph}}$. As can be seen in Fig. 5, $C_{\mathrm{ph}, \mathrm{H}}+C_{\mathrm{el}}+C_{\mathrm{D}}$ is in excellent agreement with our total $C_{P}$ result from PPMS measurements, and also in very good agreement with our $C_{P}$ result from DSC (the slight downturn in the DSC data at high temperatures is likely due to surface oxidation). This indicates that there is little intrinsic anharmonicity of phonons, that is, little $T$ dependence of phonons at constant $V$. Thus the quasiharmonic model captures the $T$ dependence of the heat capacity satisfactorily, as it did for the phonon energies. 


\section{DISCUSSION}

\section{A. Thermodynamic properties}

We first consider the possible causes for the observed stiffening of the phonons upon the introduction of vacancies. The atomic masses of $\mathrm{La}$ and Te are very close, $M_{\mathrm{La}} / M_{\mathrm{Te}}=1.09$, and for a maximum of $1 / 9 \mathrm{La}$ atoms vacant, the average mass change (per atom) is less than $0.3 \%$. Since phonon frequencies scale with mass as $\omega \sim M^{-1 / 2}$, the phonon frequency increase from the mass effect alone is less than $0.15 \%$. This is clearly insufficient to explain the observed stiffenings of $12 \%$ at the acoustic peak and $5 \%$ for the optical peaks (and overall stiffening of $\langle E\rangle$ of about $5 \%$ ). There is also only a very limited change in the lattice parameter of the system upon introduction of La vacancies, insufficient to explain the stiffening observed. Pardo et al. reported lattice parameters $a=9.634 \AA$ for $\mathrm{La}_{3} \mathrm{Te}_{4}$ and $a=9.627 \AA$ for $\mathrm{La}_{2.66} \mathrm{Te}_{4} \cdot{ }^{29}$ This decrease in lattice parameter would produce a stiffening of phonons of about $0.4 \%$ (for $\bar{\gamma}_{\mathrm{G}}=1.76$ ). Thus, a change in the force constants, corresponding to a change in the underlying electronic structure upon removal of La atoms, must be accounted for to explain the stiffening of the phonon DOS.

The change in bonding upon introduction of vacancies can be understood from the electronic structure of $\mathrm{La}_{3} \mathrm{Te}_{4}$. The introduction of La vacancies effectively removes electrons and shifts the position of the Fermi level. Firstprinciples calculations of the electronic DOS of $\mathrm{La}_{3} \mathrm{Te}_{4}$ have found a sharp peak at the Fermi level associated with two heavy electron bands with minimums at -0.18 and $-0.15 \mathrm{eV}$ below the Fermi level. ${ }^{27}$ Upon removal of $0.32 \mathrm{La}$ atoms, the Fermi level is shifted down by $-0.33 \mathrm{eV}$, into the light electron band. Consequently, analysis of the calculated band structure suggests there is a reduction in almost an order of magnitude in the density at the Fermi level: $N\left(E_{\mathrm{F}}\right)=1.046$ states $/ \mathrm{eV} /$ atom for $\mathrm{La}_{3} \mathrm{Te}_{4}$ while $N\left(E_{\mathrm{F}}\right)$ $=0.125$ states $/ \mathrm{eV} /$ atom for $\mathrm{La}_{2.68} \mathrm{Te}_{4}$ (including spin degeneracy). This large reduction in $N\left(E_{\mathrm{F}}\right)$ was also observed in our low-temperature calorimetry measurements, with a reduction by a factor of about 8 in $\mathrm{La}_{2.82} \mathrm{Te}_{4}$ (and also a large reduction in $\left.\mathrm{La}_{2.68} \mathrm{Te}_{4}\right)$. It is expected that this large reduction in the number of electrons at the Fermi level (predicted from first-principles calculations and observed with calorimetry) will affect bonding, leading to a less metallic (more ionic) system upon the introduction of vacancies.

The reduction in $N\left(E_{\mathrm{F}}\right)$ is also expected to decrease the screening of the phonon disturbances in the lattice. ${ }^{30-32}$ The electron-phonon coupling in the stoichiometric material is relatively strong in order to induce superconductivity at $T_{c}=4 \mathrm{~K}$. Since the electron-phonon coupling constant $\lambda$ is directly proportional to $N\left(E_{\mathrm{F}}\right),{ }^{30}$ a strong decrease in the electron-phonon coupling strength must result from the introduction of $\mathrm{La}$ vacancies and the resulting decrease in $N\left(E_{\mathrm{F}}\right)$. This reduced screening could explain the effective stiffening of phonons observed in the material with La vacancies. It has been observed in other BCS superconductors that acoustic modes are most affected by the electron-phonon coupling above $T_{c}{ }^{31,33,34}$ This is consistent with our neutronscattering and calorimetry measurements on $\mathrm{La}_{3-x} \mathrm{Te}_{4}$, which indicate a larger stiffening for the acoustic modes. Also, the adiabatic electron-phonon coupling was shown to affect phonon energies at temperatures much above $\theta_{\mathrm{D}}$ in other systems. ${ }^{31,32}$ This could also be the case in $\mathrm{La}_{3-x} \mathrm{Te}_{4}$ since the samples with vacancies remain stiffer than the vacancy-free $\mathrm{La}_{3} \mathrm{Te}_{4}$ at temperatures up to $780 \mathrm{~K}$.

The increase in phonon energies upon removal of La leads to a decrease in the phonon entropy and the phonon heat capacity. From the phonon DOS measured at $300 \mathrm{~K}$, we obtain a difference in vibrational entropy for the introduction of 0.32 La vacancies: $S_{\mathrm{ph}}(x=0.32)-S_{\mathrm{ph}}(x=0)$ $=-0.13 \pm 0.04 k_{\mathrm{B}} /$ at., corresponding to $4.4 \%$ vacant sites overall. Equivalently, this represents a negative phonon entropy of vacancy formation, $\Delta S_{\mathrm{ph}}^{\mathrm{v}} \mathrm{a}(300 \mathrm{~K})$ $=-2.9 \pm 0.9 k_{\mathrm{B}} /$ at. $/$ vacancy. The vibrational entropy of $\mathrm{La}$ vacancy formation is large and presents little temperature dependence within our experimental error bars. At 520 and $780 \mathrm{~K}$, we find: $\Delta S_{\mathrm{ph}}^{\mathrm{v} L a}(520 \mathrm{~K})=-2.8 \pm 1.0 k_{\mathrm{B}} /$ at. $/$ vacancy, $\Delta S_{\mathrm{ph}}^{\mathrm{V} L a}(780 \mathrm{~K})=-3.4 \pm 1.5 k_{\mathrm{B}} /$ at. $/$ vacancy. This can be compared to the configurational entropy gain associated to the introduction of vacancies. Neglecting correlations in vacancy distributions on the La sublattice, we can estimate the $\Delta S_{c f}(x=0.32)=0.34 k_{\mathrm{B}} /$ at., $\quad$ and $\Delta S_{c f}(x=0.18)=0.23 k_{\mathrm{B}} /$ at. Thus, the phonon entropy contribution significantly decreases the overall entropy change upon introduction of vacancies. The decrease in the electron density at the Fermi level with introduction of La vacancies also leads to a reduction in entropy. From the electronic DOS reported in Ref. 27, we computed $S_{\mathrm{el}}(300 \mathrm{~K})=0.090 k_{\mathrm{B}} /$ at., and since $S_{\mathrm{el}}$ is nearly proportional to $N\left(E_{\mathrm{F}}\right)$ (although at high $T$, the energy dependence of $N(E)$ may introduce corrections), the reduction in $N\left(E_{\mathrm{F}}\right)$ derived in the rigid-band approximation (see above) for $x=0.32$ gives $\Delta S_{\mathrm{el}}(300 \mathrm{~K})$ $=-0.076 k_{\mathrm{B}}$ /at., further reducing the configurational entropy gain. According to this analysis, the total entropy of La vacancy formation is still positive, however, and we estimate: $\Delta S_{\text {tot }}^{\mathrm{V} L a}(300 \mathrm{~K})=+3.0 \pm 0.9 k_{\mathrm{B}} /$ at. $/$ vacancy.

We have shown that the temperature dependence of the average phonon energy $\langle E\rangle$ in $\mathrm{La}_{3-x} \mathrm{Te}_{4}$, and of the heat capacity of $\mathrm{La}_{3} \mathrm{Te}_{4}$, can be well accounted for by the quasiharmonic model, at least for $T \geq 300 \mathrm{~K}$. The quasiharmonic model does not imply that the phonons are purely harmonic, otherwise phonons would not change at all with increasing $T$ (or $V$ ), and the total phonon contribution to the heat capacity would saturate at $3 k_{\mathrm{B}}$ /atom. However, very anharmonic systems typically display heat capacities or $T$ dependencies for phonons that deviate substantially from the quasiharmonic prediction. ${ }^{23,28}$ We thus conclude that $\mathrm{La}_{3} \mathrm{Te}_{4}$ is not in itself a particularly anharmonic material. It is still possible that a few modes would be more anharmonic than the behavior observed here but they would have to be in small enough number that they would not affect the heat capacity or the phonon DOS.

\section{B. Transport properties}

Our neutron-scattering data show that the introduction of a large number of vacancies on the La sites leads to a marked broadening of the phonons DOS (cf. Fig. 1), which can be 
interpreted as phonon scattering by point defects leading to a reduction in phonon lifetimes (and broadening in energy of their linewidths). This broadening does appear to increase slightly with the increase in vacancy concentration but the amount of broadening does not seem to depend on temperature (see Fig. 2). Thus, the point-defect scattering mechanism appears to be relatively independent of temperature, which is consistent with the behavior of the high-temperature thermal conductivity. ${ }^{2}$ We note that a redistribution of phonon energies (independent of phonon broadening), upon introduction of vacancies, cannot be ruled out on the basis of our phonon DOS measurements alone. However, the scattering of phonons by vacancies inferred from transport measurements would indicate that the phonons, even if redistributed in energy, should still exhibit a broadening from reduced phonon lifetimes.

Using the quality factor $\mathcal{Q} \simeq 10$ estimated from the broadening of the phonon DOS in $\mathrm{La}_{2.68} \mathrm{Te}_{4}$, we can estimate the lattice thermal conductivity $\kappa_{l}=\frac{1}{3} C_{\mathrm{ph}} v l=\frac{1}{3} C_{\mathrm{ph}} v^{2} \mathcal{Q} / \nu$, where $l$ is the phonon mean-free path, and $v$ is the phonon velocity, and $\nu$ its frequency. We consider that the phonon thermal transport occurs mainly through the acoustic phonons, and we use a characteristic acoustic-phonon velocity $v_{\mathrm{D}}$ determined from $\frac{3}{v_{\mathrm{D}}^{3}}=\frac{1}{v_{\mathrm{L}}^{3}}+\frac{2}{v_{\mathrm{T}}^{3}}$, with the acoustic transverse $\left(v_{\mathrm{T}}\right)$ and longitudinal $\left(v_{\mathrm{L}}\right)$ sound velocities reported for $\mathrm{La}_{2.81} \mathrm{Te}_{4}$ in Ref. 2. This gives $v_{\mathrm{D}}=2237 \mathrm{~ms}^{-1}$. We consider the acousticphonon frequency $\nu=2 \mathrm{THz}$ (corresponding to $E=8.3 \mathrm{meV}$, at the maximum of the low-energy peak of the phonon DOS), and we use the phonon heat capacity from this work $\left(C_{\mathrm{ph}} \simeq 3.1 k_{\mathrm{B}} /\right.$ atom at $\left.300 \mathrm{~K}\right)$, and the experimental lattice parameter $a=9.627 \AA$. From $\nu$ and $\mathcal{Q}$, we estimate $l \simeq 10 \mathrm{~nm}$ and $\kappa_{l} \simeq 2.7 \mathrm{~W} \mathrm{~m}^{-1} \mathrm{~K}^{-1}$ for $\mathrm{La}_{2.68} \mathrm{Te}_{4}$. This value is about four times larger than obtained from transport measurements (see Table I).

Several factors could explain this discrepancy. First, it is difficult to pinpoint which phonons are responsible for most of the lattice thermal conductivity. It is possible that grain boundaries and other defects in these mechanically alloyed materials enhance scattering of fast, long-wavelength acoustic phonons, leaving most of the conduction to occur through higher frequency, slower-propagating phonons (lower group velocities). If this is the case, our estimate of $v$ and $l$ could be too high. Since $\kappa_{l}$ varies like $v^{2}$ (for a fixed collision rate $\tau$ ), a factor of 2 in the velocity $v$ of heat-carrying phonons would bring $\kappa_{l}$ in better agreement with the transport measurements.

Also, our estimate of the phonon quality factor $\mathcal{Q}$ is an upper bound since phonons in the stoichiometric material $\mathrm{La}_{3} \mathrm{Te}_{4}$ (whose phonon DOS was convolved with the damped phonon linewidth) already have finite values of $\mathcal{Q}$. If phonons were perfectly harmonic in the reference system $(\mathcal{Q} \rightarrow \infty)$, and if our measurements had an ideal energy resolution, we would estimate larger phonon linewidths $(\Gamma \simeq E / \mathcal{Q})$ for the material with vacancies (smaller $\mathcal{Q})$. However, the quality factor we estimated is already small, and we do not expect $\mathcal{Q}$ to be less than 5 [cf Fig. 1(b)], corresponding to damping of vibration modes in about 5 oscillation periods. This would at most bring the calculated $\kappa_{l}$ down by a factor of 2 and would still indicate that the veloc- ity of heat-carrying phonons must be about 1.5 times slower than the average acoustic sound velocity. Thus, we point out that heat conduction in mechanically alloyed $\mathrm{La}_{3-x} \mathrm{Te}_{4}$ may be predominantly through relatively slow phonons, with $v \sim 1100-1500 \mathrm{~ms}^{-1}$.

It is possible that the electron-phonon coupling can have a contribution to phonon scattering in the $\mathrm{La}_{3} \mathrm{Te}_{4}$, where the electron-phonon coupling is significant. However, we expect that the electron-phonon coupling strength should be significantly reduced in the material doped with vacancies because of the large decrease in $N\left(E_{\mathrm{F}}\right)$. Nevertheless, inhomogeneities in local electronic environments in the system could lead to variations in the electron-phonon scattering in the material with vacancies.

The lack of dependence of the phonon DOS broadening on temperature (and, to a lesser extent, the good agreement with the quasiharmonic model) indicates that phononphonon scattering (associated to anharmonic components of interatomic potentials) is less important than scattering by $\mathrm{La}$ vacancies, at least for $T \leq 780 \mathrm{~K}$. Phonon-phonon scattering events become more frequent as temperature increases, however, and may become a significant source of thermal resistivity at higher temperatures. ${ }^{23,35}$

\section{CONCLUSIONS}

We have measured the phonon density of states of $\mathrm{La}_{3-x} \mathrm{Te}_{4}(x=0.0,0.18,0.32)$ compounds at 300, 520, and $780 \mathrm{~K}$, using INS, and performed calorimetry measurements in the temperature range $1.85 \leq T \leq 1200 \mathrm{~K}$. We found that the introduction of vacancies on the La sites leads to a significant broadening of phonons and an overall stiffening of phonon energies. The stiffening of the DOS was interpreted in terms of the change in bonding and screening associated with the depletion of the number of electrons at the Fermi level upon removing La atoms. The important broadening of features in the phonon DOS indicates that La vacancies are effective at scattering the phonons, confirming previous thermal transport measurements. The good agreement of the quasiharmonic model with the temperature dependence of the phonon DOS (with and without vacancies) and the heat capacity indicates the interatomic potentials are not strongly anharmonic in this material, and that the scattering by point defects is relatively insensitive to temperature.

\section{ACKNOWLEDGMENTS}

We thank David Singh for providing us with the numerical data for the electronic DOS of $\mathrm{La}_{3} \mathrm{Te}_{4}$ published in Ref. 27, and for helpful discussions. We thank Rebecca A. Mills for help with the neutron-scattering furnace. This work was partially supported by the Division of Materials Science and Engineering, Basic Energy Sciences, U.S. DOE. Work performed at the California Institute of Technology was done with the assistance of the Jet Propulsion Laboratory, under a contract with the National Aeronautics and Space Administration. The Research at Oak Ridge National Laboratory's Spallation Neutron Source was sponsored by the Scientific 
User Facilities Division, Office of Basic Energy Sciences, U.S. DOE. High-temperature calorimetry measurements were conducted at Oak Ridge National Laboratory's High Temperature Materials Laboratory, sponsored by the U. S.
Department of Energy, Office of Energy Efficiency and Renewable Energy, Vehicle Technologies Program. This work benefitted from DANSE software developed under NSF under Award No. DMR-0520547.
${ }^{1}$ G. S. Nolas, J. Sharp, and J. Goldsmid, Thermoelectrics, Basic Principles and New Materials Developments (Springer, New York, 2001).

${ }^{2}$ A. F. May, J.-P. Fleurial, and G. J. Snyder, Phys. Rev. B 78, 125205 (2008).

${ }^{3}$ M. Cutler, J. F. Leavy, and R. L. Fitzpatrick, Phys. Rev. 133, A1143 (1964).

${ }^{4}$ M. Cutler and J. F. Leavy, Phys. Rev. 133, A1153 (1964).

${ }^{5}$ M. Cutler, R. L. Fitzpatrick, and J. F. Leavy, J. Phys. Chem. Solids 24, 319 (1963).

${ }^{6}$ V. P. Zhuze, V. M. Sergeeva, and O. A. Golikova, Sov. Phys. Solid State 11, 2071 (1970).

${ }^{7}$ G. G. Gadzhiev, S. M. Ismailov, K. K. Abdullaev, M. M. Khamidov, and Z. M. Omarov, High Temp. 39, 407 (2001).

${ }^{8}$ R. M. Bozorth, F. Holtzberg, and S. Methfessel, Phys. Rev. Lett. 14, 952 (1965)

${ }^{9}$ R. N. Shelton, A. R. Moodenbaugh, P. D. Denier, and B. T. Matthias, Mater. Res. Bull. 10, 1111 (1975).

${ }^{10}$ K. Westerholt, F. Timmer, and H. Bach, Phys. Rev. B 32, 2985 (1985).

${ }^{11}$ C. Wood, Rep. Prog. Phys. 51, 459 (1988).

${ }^{12}$ A. I. Boukai, Y. Bunimovich, J. Tahir-Kheli, J.-K. Yu, W. A. Goddard III, and J. R. Heath, Nature (London) 451, 168 (2008).

${ }^{13}$ M. Christensen, A. B. Abrahamsen, N. B. Christensen, F. Juranyi, N. H. Andersen, K. Lefmann, J. Andreasson, C. R. H. Bahl, and B. B. Iversen, Nature Mater. 7, 811 (2008).

${ }^{14}$ M. M. Koza, M. R. Johnson, R. Viennois, H. Mutka, L. Girard, and D. Ravot, Nature Mater. 7, 805 (2008).

${ }^{15}$ W. Schweika, R. P. Hermann, M. Prager, J. Persson, and V. Keppens, Phys. Rev. Lett. 99, 125501 (2007).

${ }^{16}$ A. Béraud, J. Kulda, I. Yonenaga, M. Foret, B. Salce, and E. Courtens, Physica B (Amsterdam) 350, 254 (2004).

${ }^{17}$ G. Chen, M. S. Dresselhaus, G. Dresselhaus, J.-P. Fleurial, and T. Caillat, Int. Mater. Rev. 48, 45 (2003).

${ }^{18}$ M. Rotter, P. Rogl, A. Grytsiv, W. Wolf, M. Krisch, and A. Mirone, Phys. Rev. B 77, 144301 (2008).
${ }^{19}$ W. L. Cox, H. Steinfink, and W. F. Bradley, Inorg. Chem. 5, 318 (1966).

${ }^{20}$ S. W. Lovesey, Theory of Neutron Scattering from Condensed Matter (Clarendon, Oxford, 1984), Vol. 1, p. 301.

${ }^{21} \mathrm{G}$. L. Squires, Introduction to the Theory of Thermal Neutron Scattering (Cambridge University Press, Cambridge, 1978).

${ }^{22}$ M. Kresch, O. Delaire, R. Stevens, J. Y. Y. Lin, and B. Fultz, Phys. Rev. B 75, 104301 (2007).

${ }^{23}$ G. Grimvall, Thermophysical Properties of Materials (NorthHolland, Amsterdam, 1999).

${ }^{24}$ R. G. Mitarov, V. V. Tikhonov, L. N. Vasil'ev, V. S. Oskotskii, A. V. Golubkov, and I. A. Smirnov, Sov. Phys. Solid State 17, 310 (1975).

${ }^{25}$ E. Bucher, J. P. Maita, G. W. Hull, Jr., L. D. Longinotti, B. Lüthi, and P. S. Wang, Z. Phys. B 25, 41 (1976).

${ }^{26}$ V. V. Tikhonov, V. N. Bystrova, R. G. Mitarov, and I. A. Smirnov, Sov. Phys. Solid State 17, 795 (1975).

${ }^{27}$ A. F. May, D. J. Singh, and G. J. Snyder, Phys. Rev. B 79, 153101 (2009).

${ }^{28}$ D. C. Wallace, Statistical Physics of Crystals and Liquids (World Scientific, Singapore, 2002).

${ }^{29}$ M. P. Pardo, J. Flahaut, and L. Domange, Acad. Sci., Paris, C. R. 256, 1793 (1963).

${ }^{30} \mathrm{G}$. Grimvall, The Electron-Phonon Interaction in Metals (NorthHolland, Amsterdam, 1981).

${ }^{31}$ O. Delaire, M. S. Lucas, J. A. Muñoz, M. Kresch, and B. Fultz, Phys. Rev. Lett. 101, 105504 (2008).

${ }^{32}$ O. Delaire, M. Kresch, J. A. Muñoz, M. S. Lucas, J. Y. Y. Lin, and B. Fultz, Phys. Rev. B 77, 214112 (2008).

${ }^{33}$ L. Pintschovius, H. Takei, and N. Toyota, Phys. Rev. Lett. 54, 1260 (1985).

${ }^{34}$ B. P. Schweiss, B. Renker, E. Schneider, and W. Reichardt, in Superconductivity in $d$ - and f-band metals, edited by D. H. Douglass (Plenum, New York, 1976), p. 189.

${ }^{35}$ J. M. Ziman, Electrons and Phonons (Clarendon, Oxford, 1960). 